\section{Analysis of Printed Paper via Micro-ATR Mapping}

Peter Troost' ${ }^{2}$, John W. Hellgeth', David W. Schiering ${ }^{2}$, and Thomas J. Tague, J. ${ }^{2 *}$

${ }^{1}$ SRN Company, LLC, 22 Arnoldale Road, West Hartford, CT 06119

${ }^{2}$ Spectra-Tech, Inc., 2 Research Drive, Shelton, CT 06484

* Author to which correspondence should be addressed

\section{ABSTRACT}

The surface of printed paper has been examined through a fully automated attenuated total reflection (ATR) mapping technique. Automated z-axis control was utilized to bring the sample into contact with the ATR objective in a reproducible manner and an integrated mapping stage was used to position the sample. A force sensing device, the Contact Alert, was used to ensure optimal contact between the sample and internal reflection element. Global manipulations of the resultant data revealed compositional differences attributed to the printed ink, carbonate and cellulose distributions. These differences were clearly evident in the infrared, but not discernable in the visible. The bulk filler Kaolinite was identified as a post processing contaminate as well as a trace mixture of Dioctyl Phthalate and an aromatic acrylate.

\section{INTRODUCTION}

Infrared microscopes were first introduced into the marketplace more than forty years ago. Over the last fifteen years, the infrared microscope has evolved from a simple "in-compartment" single function tool to a versatile accessory capable of analyzing almost any sample. Typically, techniques for data collection include transmission, external or specular reflection, reflection-absorption, diffuse reflectance, and attenuated total reflectance (ATR) for state-of-the-art microscope products. Reflection modes of acquisition are usually preferred for rapid analysis of most sample types, because sample preparation is not required.

ATR microspectroscopy is highly favored for sample analysis for several reasons. First, sample preparation is minimized by the nature of attenuated total reflectance spectroscopy. The internal reflection crystal is brought into contact with the sample and spectra are collected from the surface of the material to be analyzed. Therefore, the surfaces of large and/or irregularly-shaped samples can be investigated easily without modification. Second, optically dense samples can be analyzed readily because the resultant evanescent wave only penetrates to a depth of about a micron into the sample [dependent on the internal reflection element (IRE)]. Third, the ATR analysis is non-destructive even for intractable samples. Examples of such samples include highly carbon-filled rubbers, printed paper and coatings.

The greatest benefit of infrared microscopy, however, is the ability to

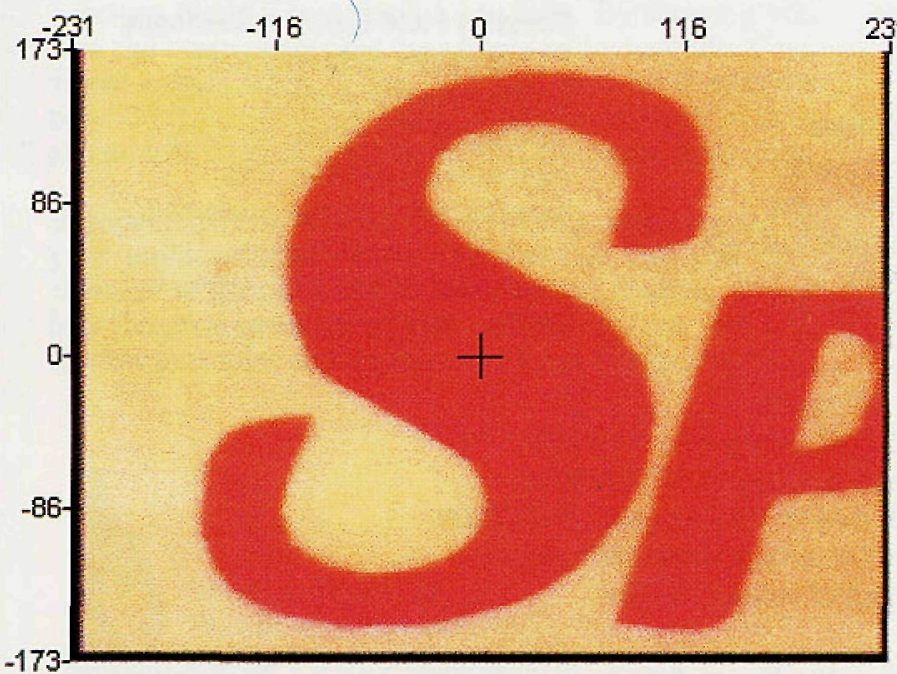

Figure 1: Viedo image of lettering printed upon a typical business card. The region of analysis includes the " $S$ " and its surrounding paper surface. Axes are given in arbitrary units. spatially discriminate regions or domains within a sample. With recent developments in the integration of software and hardware, automated sample analysis may be accomplished for the first time with relative ease. Software control of the $x$ and $y$ axes via mapping stage and $z$ focus through autofocus permits automated microanalysis of large samples. This automated acquisition capability is applicable to many areas including contaminant analysis of semiconductors, thin layer laminate analysis, and concentration gradient of pharmaceutical products. This level of automation, in combination with microscopic ATR techniques, yields exceptional potential for automated surface analysis on a large sample scale.

The purpose of this investigation was to demonstrate the utility of ATR mapping for determining the character and quality of paper surface treatments and printing inks. A typical business card with raised letter printing was mapped in an effort to determine the nature of the distribution of carbonate as well as the chemical composition and distribution of the printed ink.

\section{EXPERIMENTAL}

Printed paper products were examined with an IRus II infrared microscope operated in the reflection mode and equipped with a Spectra-Tech, Inc. (STI) patented ATR objective and a Nicolet Instrument Corporation mid-band $0.25 \mathrm{~mm}$ MCT detector. The ATR objective was fitted with a curved zinc selenide internal reflection element, which permitted viewing of the sample for easy setup of the experiment. Samples were placed upon a $2^{\prime \prime}$ by $5^{\prime \prime} X-Y$ motorized stage (STI) which has a positional accuracy of better than 1 micron. Z-axis positioning of the stage was obtained through an Autofocus control accessory (STI) placed upon the mechanical coarse/fine focus control of the scope. The data collection, stage and z-axis motion control, as well as data manipulation were controlled through Nicolet's OMNIC software with the Atlus infrared microscope mapping package. Initial sample positioning was obtained through the use of viewing oculars as well as a video camera. Video images at 468 by 512 pixel resolution were obtained via Video Capture Board (STI) mounted into the microscope's control computer.

Mapped spectra were obtained from 4.2 by $5.0 \mathrm{~mm}$ square areas through repetitive sample contact with the zinc selenide IRE of the ATR objective. Data was acquired with a remote aperture that yielded redundant image masking of a 37 microns spot at the IRE contact zone. Spectral maps were generated from

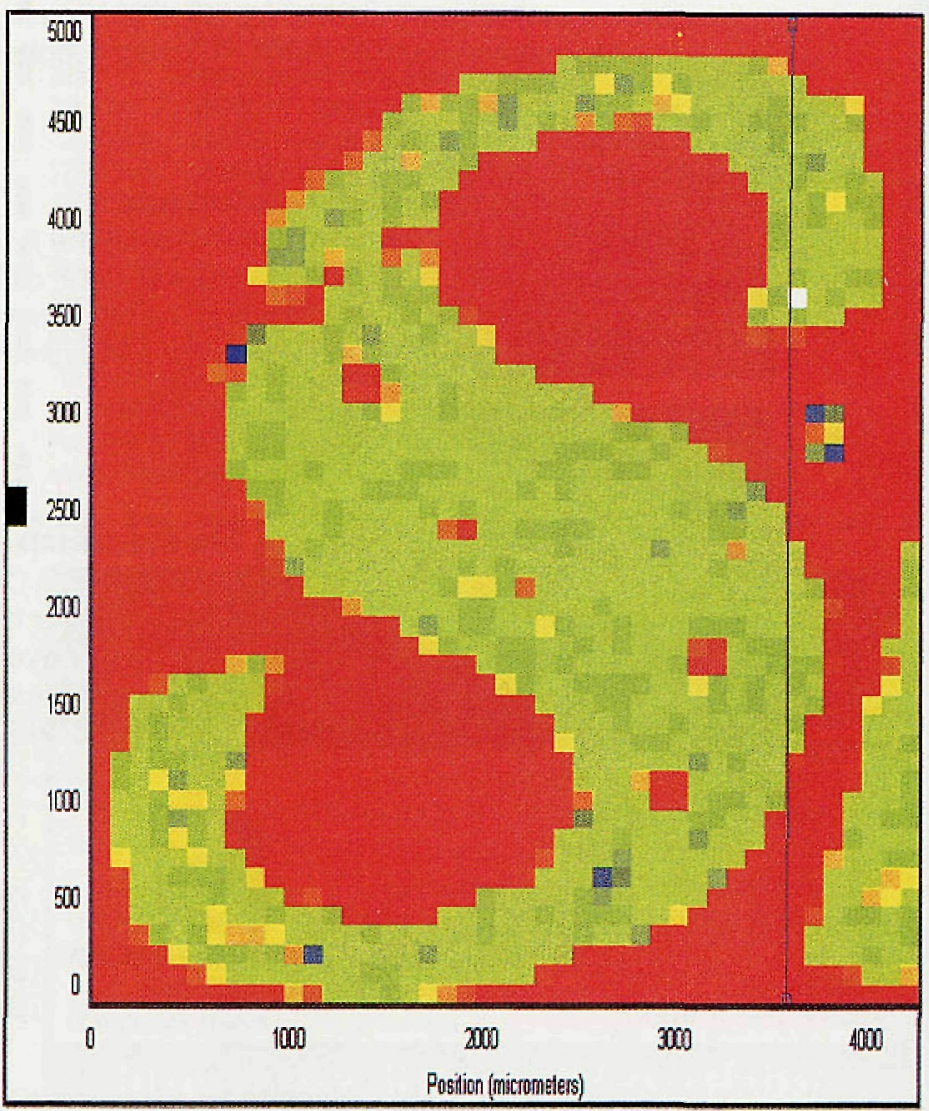

Figure 2: Total infrared absorbance map for 5000 by 4000 micrometer regions of printed business card. 
point spacing of 100 microns across the sample surface (2200 points/map). Initial Z-axis position for contact was determined by viewing contact directly through the ATR objective. Constant force of contact was monitored throughout the mapping process with a Contact Alert System (STI). All spectra were collected at 8 wavenumbers resolution using 2 scans coadded and Fourier processed with Mertz phase correction and Happ-Genzel apodization. This yielded point measurement times of 0.7 seconds. Post-run data manipulation involved ratioing to a background of 16 scans co-added, conversion to $\log (1 / \mathrm{R})$ and baseline correction. All spectral data manipulations were performed globally across the entire spectral data set.

\section{RESULTS AND DISCUSSION}

The purpose of this study was to demonstrate the utility of micro-ATR mapping for determining the character and quality of paper surface treatments and printing inks. The sample examined was a typical business card with raised letter printing. Particular issues of interest were the consistency of the paper surface with respect to the distribution of carbonate and the character and distribution of the printing ink.

Spectral imaging through infrared microspectroscopy yields an exceptional amount of information with the ability to collect and archive both visual and spectral images. As an example, the area of interest on the sample is shown in the video capture image given in Figure 1. This video image was acquired with low magnification $(10 x)$ to capture the complete map region. It visually defines the areas of paper and inked surfaces as well as any visible defects. The complementary infrared spectral map is presented in Figure 2. This false color image represents the total change in infrared intensity across the spectral range of 4000 - 650 wavenumbers. It is comprised from spectra taken at $2193 x$, y coordinate pair map positions. This image clearly delineates regions of ink and paper as well as potential defect sites which could only be determined by infrared spectroscopy.

In the OMNIC/Atlus ${ }^{\text {TM }}$ software package, graphical tools permit easy manipulation of mapped spectral data to reveal specific chemical information. Spectra from a specific position within the map are accessed through the use of cursor

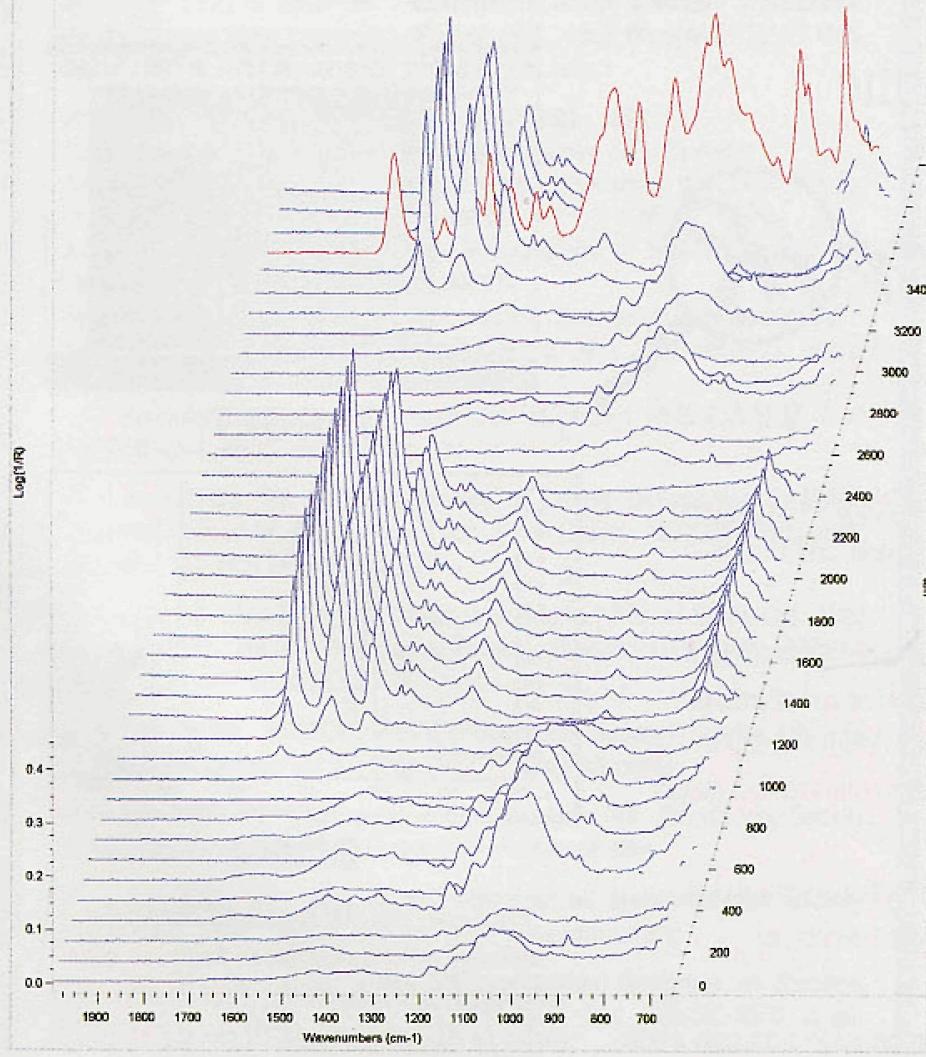

Figure 3: Spectra obtained along the annotated profile line (shown in Figure 2) which indicate transitions from paper to ink locations. and line tools. In the infrared spectral image shown, transitions between the paper and print surfaces are indicated along the annotated profile line. In Figure 3 , the compositional transitions from zones of paper and ink are indicated in a plot of absorbance versus distance versus wavenumbers and easily relate the specific changes in chemical composition along that longitudinal line. The spectra clearly show the distribution of three species; cellulose, an amide based ink and an occasional carbonate species. In these spectra, cellulose is indicated by a broad absorption band centered at 1050 wavenumbers. The amide based print ink is revealed in the spectra containing amide bands at 1636 and 1539 wavenumbers and is easily distinguished from the spectra of cellulose. A carbonate species appears randomly in several of the cellulose spectra. It appears as a broad absorption band centered at 1390 wavenumbers and a sharp absorption band at 867 wavenumbers. Figure 4 shows the individual spectra of the pertinent species.

The top spectrum in Figure 5, also the fourth spectrum from the top in Figure 3 , reveals the presence of a localized defect on the surface of the print ink. Visual inspection of the sample failed to reveal its presence. A band specific profile of the $1730 \mathrm{~cm}^{-1}$ band, shown in Figure 6 accentuates this defect. Quick analysis of the raw spectrum reveals that kaolinite, a common filler used in the paper manufacturing process, is present. Spectral subtraction of Kaolinite, second from the top in Figure 5, from the raw spectrum was performed yielding the spectrum located third from the top. Spectral searching on the resultant spectrum indicated the presence of dioctyl phthalate and an aromatic acrylate. Although this assignment is tentative, dioctal phthalate and aromatic acrylates are used in the paper manufacturing process

\section{Figures continued on following page}

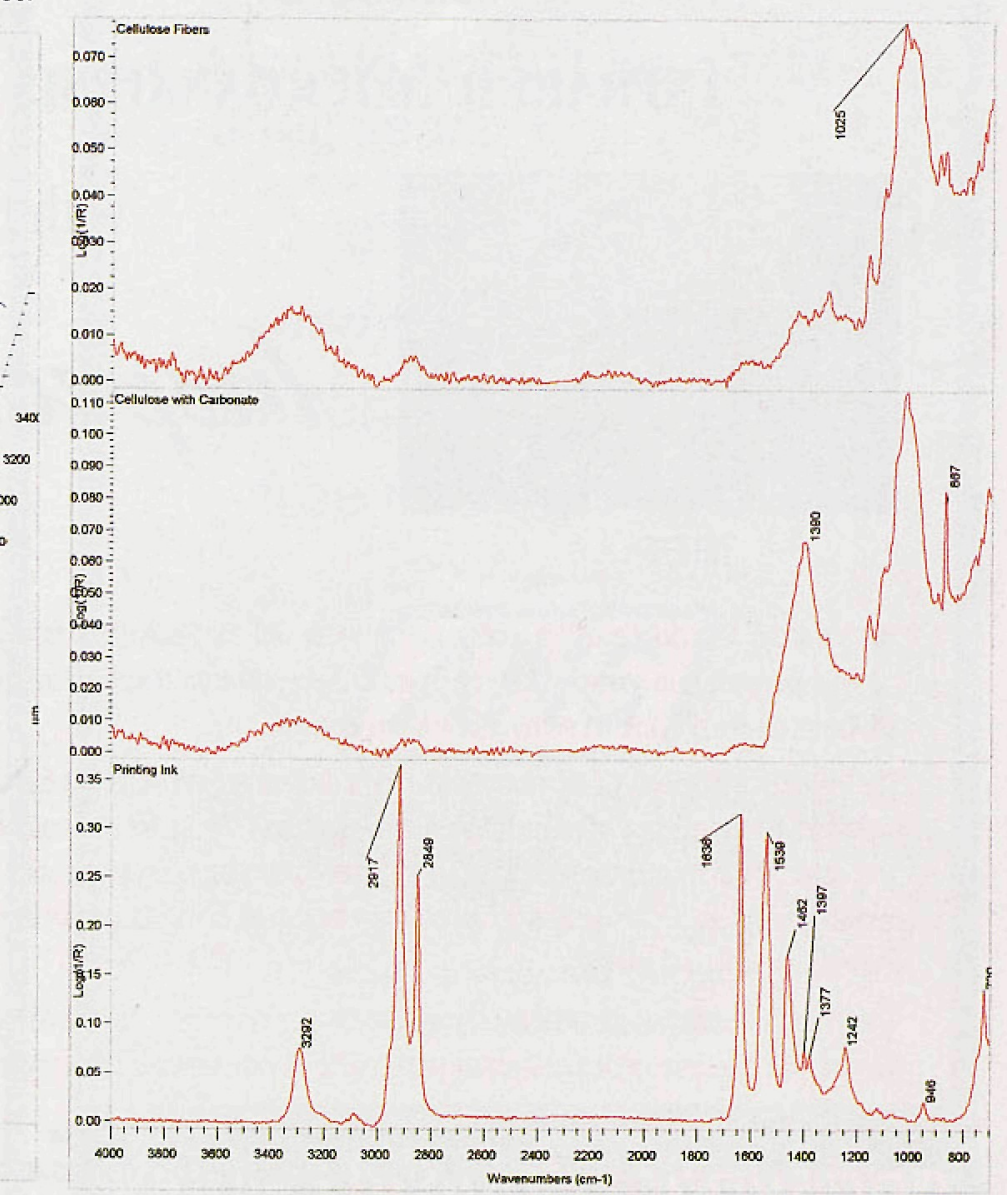

Figure 4: Spectra obtained from specific regions indicating cellkulose, carbonate on cellulose and ink constituents. 


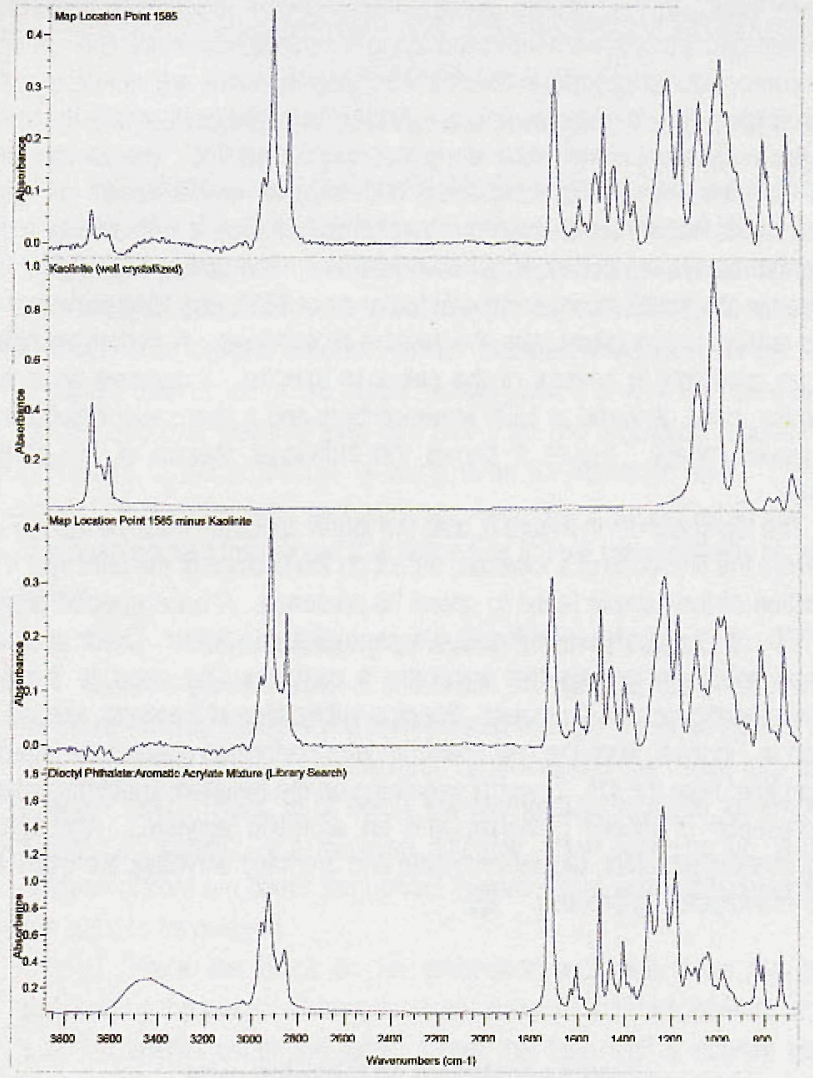

Figure 5: Top - Spectrum recorded at map location 1585. Second from top - Reference spectrum of Kaolimite. Second from bottom - map point 1585 after Kaolinite subtraction. Bottom - Reference spectrum of 1:1 Dioctyl Phthalate: Aromatic acrylate mixture.
Analysis of Printed Paper via Micro-ATR Mapping Continued from preceding page

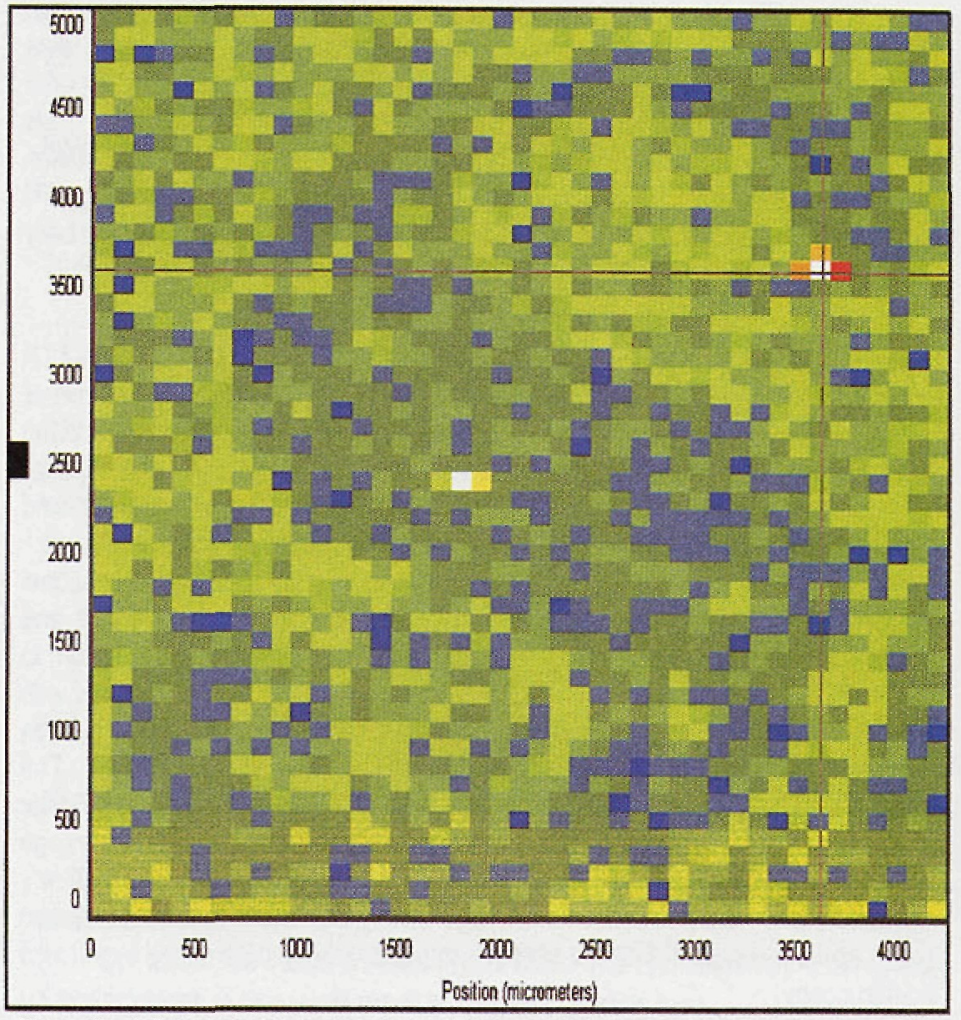

Figure 6: Infrared spectral map obtained from peak height analysis of defect-specific absorption band at 1730 wavenumbers. Locations shown in white indicatge high concentrations of contaminant.

\section{Infrared Microanalysis Just Got A Lot Easier}

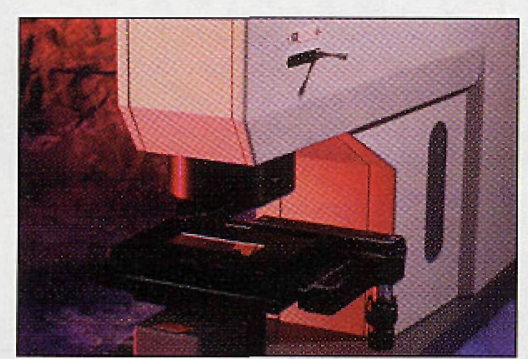

SEE

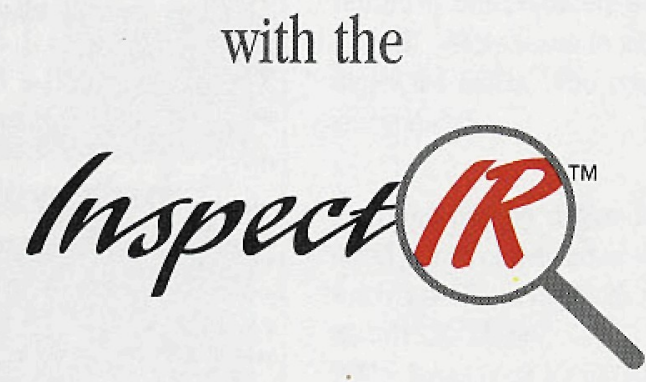

with the

t's that easy to acquire excellent quality spectral results with the InspectRT ${ }^{\mathrm{TM}}$ from Spectra-Tech. Simply
view your sample in a video window on your monitor, position the target on the sample, and analyze -
there's no need for time consuming sample preparation!

The InspectR is a unique, FT-IR microanalysis and video imaging accessory that combines Spectra-Tech's exclusive ATR and external reflection microanalysis capabilities with exceptional ease of use and affordability. The InspectIR incorporates the latest advanoes in infrared sampling technology, optical design and digital imaging to produce a microanalysis accessory for use by anyone in your laboratory to acquire excellent quality spectral data easily and quickly.

The InspectIR connects to the external beam of any FT-IR spectrometer. Its patented optical system and an integral MCT detector provide the sensitivity you need for all your routine microanalysis experiments.

\footnotetext{
SPECTRA-IECH/Nh $\begin{aligned} & 2 \text { Research Drive, Shelton, CT } 06484 \\ & \text { Phone: 800-243-9186 In CT: 203-926-8998,FAX: 203-926-8909 } \\ & \text { Look for us on the Web at: http://www.spectra-tech.com }\end{aligned}$
}

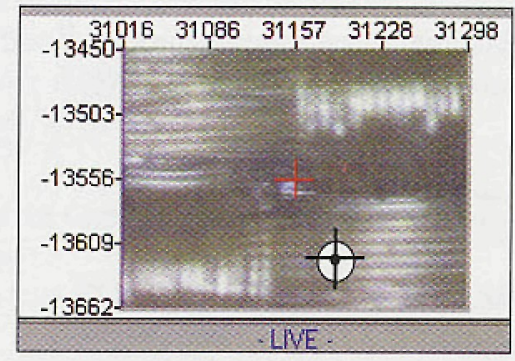

TARGET

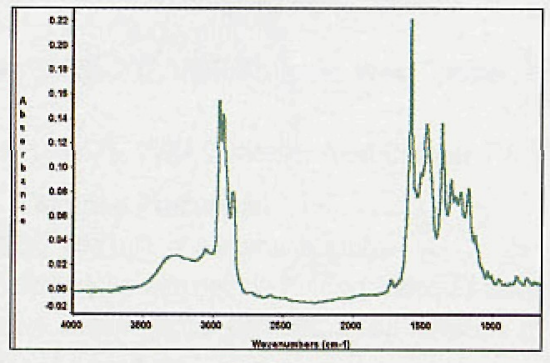

SCAN

Find out bow the Inspectir simplifies infrared micnoanalysis to improve your laboratory's productivity. Call Spectra-Tech today to discuss your FT-IR sampling needs. 